\title{
Molecular characterization of thyroid hormone receptor beta from Schistosoma japonicum and assessment of its potential as a vaccine candidate antigen against schistosomiasis in BALB/C mice
}

Chunhui Qiu ${ }^{1,2}$, Shengfa Liu ${ }^{1 *}$, Yang Hong ${ }^{2}$, Zhiqiang Fu², Meimei Wei ${ }^{1,2}$, Dezhou Ai ${ }^{2}$ and Jiaojiao Lin ${ }^{2^{*}}$

\begin{abstract}
Background: Thyroid hormones (TH) modulate growth, development and differentiation and metabolic processes by interacting with thyroid hormone receptors (THRs). The purpose of this study was to identify a novel thyroid hormone receptor beta encoding gene of Schistosoma japonicum (SjTHRß) and to investigate its potential as a vaccine candidate antigen against schistosomiasis in BALB/c mice.
\end{abstract}

Methods: The full-length CDNA sequence of SjTHR $\beta$, its gene organization, and its transcript levels were characterized, and the phylogenetic relationship between THR, RAR and RXR from other organisms were analysis, the ability of this protein binding to a conserved DNA core motif, and its potential as a vaccine candidate antigen against schistosomiasis in BALB/C mice were evaluated.

Results: The SjTHRB CDNA was cloned, verified by 5' and 3' Rapid Amplification of cDNA Ends and shown to be polyadenylated at the $3^{\prime}$ end, suggesting the transcript is full-length. SjTHRß is homologous to THRs from other species and has a predicted conservative DNA binding domain and ligand binding domain that normally characterizes these receptors. A comparative quantitative PCR analysis showed that SjTHR $\beta$ was the highest expressed in $21 \mathrm{~d}$ worms and the lowest in $7 \mathrm{~d}$ and $13 \mathrm{~d}$ schistosomula. The CDNA corresponding to DNA binding domain (SjTHRß-DBD) and ligand binding domain (SjTHRß-LBD) were cloned and subsequently expressed in $E$ coli. The expressed proteins were used to immunize mice and generate specific serum against recombinant SjTHR $\beta$ (rSjTHRB). Western blotting revealed that anti-rSjTHRß-LBD serum recognized two protein bands in extracts from $21 \mathrm{~d}$ worm with molecular sizes of approximately $95 \mathrm{kDa}$ and $72 \mathrm{kDa}$. Electrophoretic mobility shift assay (EMSA) analysis showed that $r S j T H R B-D B D$ could bind to a conserved DNA core motif. Immunization of BALB/c mice with rSjTHRB-LBD could induce partial protective efficacy(27.52\% worm reduction and $29.50 \%$ liver eggs reduction) against schistosome infection. Enzyme-linked immunosorbent assay showed that mice vaccinated with recombinant SjTHRß-LBD (rSjTHRß-LBD) generated increased levels of specific lgG, lgG1 and lgG2a antibody. Bio-plex analysis demonstrated that rSjTHRß-LBD induced considerably higher levels of T helper 1 cytokines (IL-2, IL-12 and TNF-a) than T helper 2 cytokines (IL-10, IL-4), suggesting that rSjTHRß-LBD vaccination could stimulate mixed Th1/Th2 types with Th1 dominant immune responses.

Conclusions: Our study presented here identified SjTHR $\beta$ as a new schistosome THR that might play an important role in host-parasite interaction and be a vaccine candidate for schistosomiasis.

\footnotetext{
*Correspondence: liuxie@xmu.edu.cn; JJlin@shvri.ac.cn

'School of Life Sciences, Xiamen University, Xiamen 361005 Fujian Province,

China

${ }^{2}$ Shanghai Veterinary Research Institute, Chinese Academy of Agricultural

Sciences, Key Laboratory of Animal Parasitology, Ministry of Agriculture of

China, Shanghai 200241, P R China
}

\section{Biomed Central}

(c) 2012 Qiu et al.; licensee BioMed Central Ltd. This is an Open Access article distributed under the terms of the Creative Commons Attribution License (http://creativecommons.org/licenses/by/2.0), which permits unrestricted use, distribution, and reproduction in any medium, provided the original work is properly cited. 


\section{Background}

Thyroid hormones (TH) modulate growth, development and differentiation and metabolic processes by interacting with THRs $[1,2]$. THR which belongs to nuclear receptor (NR) superfamily 1 possesses a typical nuclear receptor modular motif, including a poorly conserved $\mathrm{N}$-terminal $\mathrm{A} / \mathrm{B}$ domain, a highly conservative DNA binding domain (DBD), a hinge region and a moderately conservative Cterminal ligand binding domain [2]. In the traditional model of THR action, THR regulates gene expression through its binding to the promoter region of the target gene called specific thyroid hormone- response elements (THRE) by the DBD [3]. A number of artificial and natural THREs are a direct, inverted or everted repeat or palindrome of the DNA sequence AGGTCA. THR can bind to the THRE as a monomer, a homodimer or as a heterodimer with RXR and positively or negatively control the gene transcription and expression [4].

Schistosomiasis caused by parasitic blood flukes is a major public health problem worldwide. Approximately 200 million people are infected with pathogenic schistosomes, of which 20 million have advanced to lifethreatening disease $[5,6]$.

Schistosomes live in the vascular system of the host, where they feed on blood components and respond to molecular signals from the host to develop normally [7].

Earlier researches have revealed that schistosomes can take advantage of signals from each other and from the host to facilitate their development, however, little is known about the molecular characterization of these signals [8-10]. Previous studies suggest that the existence of THR in S. japonicum and the parasite may use an endogenous TH/THR signalling pathway for growth and development [11]. Herein, a novel mammalian orthologue of thyroid hormone receptor beta of NR superfamily 1 from S. japonicum (SjTHR $\beta$ ) was identified and characterized. Study of schistosome NR will help us to understand how they regulate signaling pathways in the schistosome itself and add to our knowledge of the molecular relationship between the schistosome and its hosts [12].

\section{Methods}

\section{Parasites and animals}

The life cycle of $S$. japonicum (Chinese mainland strain, Anhui isolate) was maintained in New Zealand rabbits and Oncomelania hupensis in Shanghai Veterinary Research Institute, Chinese Academy of Agricultural Sciences. Specific pathogen free (SPF) male BALB/c mice, 6 weeks old, were purchased from Shanghai laboratory animal center, Chinese academy of sciences (Shanghai). 7d, 13d, 21d, 28d, 35d and 42d Schistosomes were collected by perfusion of New Zealand rabbits infected percutaneously with 1000 to 6000 cercariae of S. japonicum respectively, shed from Oncomelania hupensis snails as described [13]. Animal care and all procedures involving animals were conducted according to the principles of the Shanghai Veterinary Research Institute, CAAS.

\section{Cloning of S. japonicum thyroid hormone receptor}

The total RNAs were extracted from 21d worms using Trizol reagent (Invitrogen), following the manufacturer's instructions. DNA remaining in the RNA solution was digested with DNase I (TaKaRa) and then purified by an RNeasy mini kit (Qiagen, Germany). The concentration and purity of the RNAs were evaluated by spectrophotometry (Eppendorf, German). The cDNAs were synthesized using the PrimeScriptTM RT reagent Kit (TaKaRa, China), according to the user manual. According to putative thyroid hormone receptor(predicted Sjc_0027230) sequence deposited in http://lifecenter.sgst.cn/schistosoma/cn/schdownload several pairs of primer were designed. Two correct PCR products corresponding to partial fragment of putative thyroid hormone receptor were amplified individually by primer 1 (Forward primer: 5'-AACAAACTACGAACGCAAAG-3' and reverse primer: $5^{\prime}$-TTAAAGCTACCGCACGAA-3') and primer2 (Forward primer: $5^{\prime}$-ATTATCGTGCTATGACTTG-3' and reverse primer: $5^{\prime}$-GTCTTTGCGTTCGTAGTT-3'). In order to obtain complete mRNA sequence, two pairs of RACE primers were designed based on amplified PCR sequence. After total RNAs were extracted, reverse transcription (RT) and PCR were performed according to the user's manual of the SMART ${ }^{\text {TM }}$ RACE cDNA Amplification Kit (Clontech). The transcription initiation site and the 3/-end of SjTHR $\beta$ were amplified by $5^{\prime}$-RACE and $3^{\prime}$ RACE, respectively. The $3^{\prime}$-RACE was performed with the following primers: forward gene-specific primer, $5^{\prime}$-TCACTTGGAATCGTCGAACCTA-3'; forward genespecific nested primer, 5'-GCCCAAATGATTCGTGCGGTAG-3'. The $5^{\prime}$-RACE was performed with the following primers: reverse gene-specific primer, $5^{\prime}$ CCATCCCACCGCTTATGCACCGATCAAA-3'; reverse gene-specific nested primer, 5'-GCTTATCAGAAACAGAGCAGCGACCTTG-3'; PCR products obtained from RACE were ligated with pMD19-T vector (TaKaRa) and were then transformed into DH5 $\alpha$ competent cells (Invitrogen). The positive clones were detected and sequenced. DNAStar software was employed to assemble the sequences of $3^{\prime}$-RACE, $5^{\prime}$-RACE, and mRNA into a complete cDNA sequence.

\section{Real-time PCR analysis}

The mRNA expression of SjTHR $\beta$ was evaluated in 6 different developmental stages including 7, 13, 21, 28, 35 and $42 \mathrm{~d}$ worms of S. japonicum. The cDNA products were obtained by the method described above. The primers specific to SjTHR $\beta$ (forward: 5'-AGTTCCTGAATTTGAGCTGT-3' 
and reverse: $5^{\prime}$-TTTCTTGTTGATGAGACGGC-3') can generate an amplicon of 218 bp. S. japonicum NADH-ubiquinone reductase was used as a housekeeping gene for this study [14]. Primers specific for NADH-ubiquinone reductase gene were 5'-CGAGGACCTAACAGCAGAGG$3^{\prime}$ (sense) and 5'-TCCGAACGAACT TTGAATCC-3' (antisense), and the PCR product size of the internal control is $174 \mathrm{bp}$. The PCR amplification was carried out using the reverse-transcribed cDNA as template with the SYBR Premix Ex Taq ${ }^{\mathrm{TM}}$ (TaKaRa, Japan) using the Mastercycler ep realplex4 (Eppendorf, Germany) real-time PCR detection System. The cycling protocol was as follows: $95^{\circ} \mathrm{C}$ for $10 \mathrm{~s}$ and 40 cycles of $95^{\circ} \mathrm{C}$ for $15 \mathrm{~s}, 55^{\circ} \mathrm{C}$ for $15 \mathrm{~s}, 72^{\circ} \mathrm{C}$ for $15 \mathrm{~s}$. The value of fluorescence was detected at the end of each extension step. Specificity of the PCR products was determined by melting curve analysis and agarose gel electrophoresis. Negative control reactions, which contain all of the reagents except cDNA template, were included to make sure that the reaction system was not contaminated. Each reaction was performed in triplicate the entire experiment was carried out at least three times.

\section{Gene organization}

The web-based query system (http://lifecenter.sgst.cn/ blast/cn/) was used to BLAST search the cDNA sequence. The exon/intron boundaries were determined.

\section{Phylogenetic analysis of SjTHR $\beta$}

Fifteen nuclear receptor protein sequences from different species were evaluated in the phylogenetic analysis. The phylogenetic tree was constructed from deduced amino acid sequences of SjTHR $\beta$. After alignment with CLUSTAL program in Mega4, an evolutionary tree was drawn by the neighbor-joining method.

\section{Generation of anti rSjTHR $\beta$-DBD and anti rSjTHR $\beta$-LBD specific antibodies}

DNA sequence encoding DNA binding domain (amino acid 119-250) of SjTHR $\beta$ was amplified by PCR using the forward primer 5'-GCGGAATTCCCTCCTAAAGTTAAAAAAAGAG- $3^{\prime}$ and the reverse primer $5^{\prime}$-GCGCTCGAGAGTAGTCAGTGAATCTGTAAC- $3^{\prime}$. The DBD fragment was then subcloned into the pET28a (+) expression vector between the restriction recognition sites of EcoR I ( $5^{\prime}$ end underline) and Xho I ( $3^{\prime}$ end underline). The recombinant plasmid expressed SjTHR $\beta$-DBD in E. coli BL21 (DE3). LBD sequence corresponding to the predicted LBD (amino acid 556-817) of SjTHR $\beta$ was amplified by PCR using the forward primer 5'-GCGGGATCCGATATAGACAAAGATCCTG- ${ }^{\prime}$ and the reverse primer 5'-GCGCTCGAG ATCAGTCTGCTGGATCAT-3'. The LBD fragment was subcloned into the pET32a $(+)$ expression vector between the restriction recognition sites of
BamH I ( $5^{\prime}$ end underline) and Xho I ( $3^{\prime}$ end underline). The recombinant plasmid expressed SjTHR $\beta$-LBD fusion protein in E. coli BL21 (DE3). These fusion proteins were purified by affinity chromatography on $\mathrm{Ni}^{2+}$-NTA resin. After dialysis against PBS, the purified fusion proteins of SjTHR $\beta$-DBD and SjTHR $\beta$-LBD were used to immunize $\mathrm{BALB} / \mathrm{c}$ mice. The mice were injected subcutaneously four times at two week intervals with the purified protein in Montanide ISA 206 adjuvant $(20 \mu \mathrm{g} / 100 \mu \mathrm{L} /$ mouse; Seppic). Antiserums were collected two weeks after the last injection.

\section{Western blot}

For Western blot, 21d worm antigen was prepared from worms of S. japonicum and unrelated protein was extracted from E. coli BL21 (DE3) as described (You et al. 2010). The extracts of 21d worm and unrelated protein were separated by SDS/PAGE (12\% gel) and then transferred onto nitrocellulose membrane (Whatman, Germany). The membranes were blocked with 5\% non fat milk in PBS (blocking buffer) containing 0.05\% Tween20 for $1 \mathrm{~h}$. After washing, the membrane was incubated in a 1:100 dilution of anti SjTHR $\beta$-LBD mouse serum overnight at $4^{\circ} \mathrm{C}$. Subsequently, horseradish peroxidase conjugated goat anti-mouse secondary IgG (Sigma, USA) was used to bind first antibody. Three washes with 10mins each were performed after each step. Finally, detection was performed with the $3,3^{\prime} 5,5^{\prime}$ Tetramethyl Benzidine dihydrochloride (TMB, Sigma, USA) according to the instruction of manufacturer. Image was obtained by using ImageQuant 300 Capture Imaging System (GE Healthcare, USA).

\section{EMSA}

EMSA was performed using a LightShift chemiluminescent EMSA kit (Thermo scientific, pierce, USA) with biotin-labeled oligonucleotide (Invitrogen, China), corresponding to sequence of Half-site (5'-GTACCGTAAGGTCACTCGCG-3') and DR0 (5'-CCGTAAGGTCAAGGTCACTCG-3'). The binding reactions were incubated on ice for 30 mins in $20 \mu \mathrm{L}$ reaction mixture containing $2 \mu \mathrm{L} 10 \times$ binding buffer, $10 \mu \mathrm{L}$ ultrapure water, $1 \mu \mathrm{L} 50 \%$ glycerol, $1 \mu \mathrm{L} 100 \mathrm{mM} \mathrm{MgCl} 2,1 \mu \mathrm{g}$ ploy(dI: dC), $1 \mu \mathrm{L} 1 \% \mathrm{NP}-40,2 \mu \mathrm{L}$ dialyzed rSjTHR $\beta$-DBD and $2 \mu \mathrm{L}$ biotin-probe. After binding reaction, samples were loaded onto $6.5 \%$ polyacrylamide gel in $0.5 \times$ Tris-borate- EDTA buffer and electrophoresed at $100 \mathrm{~V}$ at $4^{\circ} \mathrm{C}$ for $1 \mathrm{~h}$. Biotinlabeled, double-stranded DNA was transferred to positively charged nylon membrane (Thermo scientific, pierce, USA). After cross-linking the transferred DNA to membrane by UV-light, biotin-labeled DNA was integrated with streptavidin-horseradish peroxidase conjugate. Finally, the result was detected using enhanced chemiluminescence and the images were taken by Image Quant LAS 
4000MINI (GE, USA). For the non-specific competition experiment, the sequence (5'-CTAGTATACGTACTACTC TGA-3') was designed and annealed to its complementary oligonucleotide and then added into the binding reaction mixture. Half-site and DR0 without biotin-label were synthesized and added into the binding reaction in the specific competition experiment. For the supershift experiment, $2 \mu \mathrm{g}$ dialyzed $\mathrm{rSj}$ THR $\beta$-DBD was incubated with $1 \mu \mathrm{L}$ anti $\mathrm{rSj}$ THR $\beta$-DBD serum for 10 mins before incubation with the biotin-labeled probe.

\section{Evaluation of immune protection against $S$. japonicum challenge induced by the $r$ SjTHR $\beta$-LBD}

Thirty-six mice were randomly allotted into 3 groups of 12 mice per group and injected subcutaneously (SC) three times at 2 week intervals with 206 adjuvant (Seppic, France) in PBS (100 $\mu \mathrm{l} /$ mouse $), r S j T H R \beta-L B D$ in 206 adjuvant $(20 \mu \mathrm{g} / 100 \mu \mathrm{l} /$ mouse $)$, and PBS only (100 $\mu \mathrm{l} /$ mouse), respectively. Two weeks after the last injection, all mice were challenged with $40 \pm 2$ cercariae. The worm and egg counting reduction rates were used to evaluate the efficacy of immunization. Adult worms perfused from BALB/c mice were obtained and counted. The worm reduction rates were calculated using the formula: percentage reduction in worm burden $=($ mean worm burden of control group - mean worm burden of vaccinated group)/mean worm burden of control group $\times 100 \%$. Livers of each infected mouse were weighed and homogenized in $5 \mathrm{ml}$ of PBS, and then $5 \mathrm{ml}$ of $10 \% \mathrm{NaOH}$ was added and mixed. The mixture was incubated at $56^{\circ} \mathrm{C}$ for $45 \mathrm{~min}$ and then mixed sufficiently. $10 \mu \mathrm{l}$ mixture was used to count the number of eggs and it was performed in triplicate. The data was transformed to eggs per gram (EPG). Egg counting reduction rate was calculated by the formula (mean EPG of control group - mean EPG of vaccinated group)/ mean EPG of control group $\times 100 \%$.

\section{Detection of SjTHR $\beta$-LBD specific antibodies}

Mouse sera was collected by retro-orbital bleeding before the first vaccination, 10 days post each vaccination and before the mouse perfusion. ELISA assay was performed to detect specific IgG antibodies against rSjTHR $\beta$-LBD. rSjTHR $\beta$-LBD at a working concentration of $10 \mu \mathrm{g} / \mathrm{ml}$ ( $\mathrm{pH}$ 9.6) was coated on 96-well microtiter plates (Costar, USA) overnight at $4^{\circ} \mathrm{C}$. The wells were blocked with $5 \%$ skimmed milk in PBS containing $0.05 \%$ Tween20 (PBST) for $1 \mathrm{~h}$ at $37^{\circ} \mathrm{C}$, thereafter washed 3 times by PBST. All test sera were diluted in 1:100 in PBST, added onto plates and incubated for $1 \mathrm{hr}$. The goat anti-mouse IgG conjugated horseradish peroxidase (Sigma, USA) in 1:2500 dilutions was added to bind the primary antibody. Thorough washes were taken after each antibody incubation step. Substrate of $100 \mu \mathrm{l}$ soluble 3,3'5, 5'-tetramethyl benzidine dihydrochloride (TMB, Sigma, USA) was added to each well. The plates were incubated at $37^{\circ} \mathrm{C}$ in the dark for $10 \mathrm{~min}$. The reaction was terminated by $2 \mathrm{M}$ sulfuric acid (50 $\mu \mathrm{l} /$ well). The absorbance was read at $450 \mathrm{~nm}$ on microplate reader (BioTek, USA). For IgG1 and IgG2a detection, we used goat anti-mouse IgG1 and IgG2a conjugated horseradish peroxidase (Sigma, USA) in 1:2000 dilutions as secondary antibodies, and the other steps were the same with those described in IgG detection.

\section{Cytokine analysis}

Bio-Plex mouse cytokine assay for simultaneous quantization of interleukin (IL)-2, IL-4, IL-10, IL-12, tumor necrosis factor (TNF)- $\alpha$, interferon (IFN)- $\gamma$ was employed according to the recommended procedure. In brief, the premixed standards generated a stock concentration of $32,000 \mathrm{pg} / \mathrm{mL}$ for each cytokine. The standard stock was serially diluted in PBS to generate 9 points for the standard curve. The assay was performed in a 96-well filtration plate supplied with the assay kit. Premixed beads $(50 \mu \mathrm{L})$ coated with target capture antibodies were transferred to each well of the filter plate and washed twice with Bio-Plex wash buffer. Premixed standards or anti rSjTHR $\beta$-LBD serum $(50 \mu \mathrm{L})$ were added to each well containing washed beads. The plate was shaken for $30 \mathrm{~s}$ with $1,100 \mathrm{rmp}$ and then incubated at room temperature for $30 \mathrm{~min}$ with lower shaking speed. After incubation and 3 washes, premixed detection antibodies $(50 \mu \mathrm{L})$ were added to each well. The incubation was terminated after shaking for $10 \mathrm{~min}$ at room temperature. After 3 washes, the beads were resuspended in $125 \mu \mathrm{L}$ of Bio-Plex cytokine assay buffer. Beads were read on the Bio-Plex suspension array system, and the data was analyzed using BioPlex ManagerTM software with 5PL curve fitting.

\section{Statistical analyses}

Results in this work were acquired from at least three replicates on independent experiments with identical protocols. All values are expressed as means \pm SD. Statistical analyses were performed by Student's $t$ test, and $\mathrm{P}<0.05$ was defined as statistical significance.

\section{Results}

\section{Sequence analysis of S. japonicum thyroid hormone} receptor

Using PCR, $3^{\prime}-$ RACE and 5'- RACE, a sequence encoding SjTHR $\beta$ from $S$. japonicum cDNA was cloned. The SjTHR $\beta$ fragment was $3196 \mathrm{bp}$ in length and contained 357 bp of $5^{\prime}$-untranslated region (UTR) and 328 bp of $3^{\prime}-$ UTR. The predicted entire open reading frame (ORF) of SjTHR $\beta$ includes 836 amino acids with an approximate molecular weight of $94 \mathrm{kDa}$. NCBI BLASTP search indicated that the best match for this protein sequence was $S$. 
mansoni THR $\beta$ (GenBank accession no. AAR29359.1) with $66 \%$ identity. In addition, SjTHR $\beta$ shows $20 \%, 18 \%$ and $35 \%$ identities with Homo sapiens THR $\beta$ (HTHR $\beta)$, Branchiostoma floridae THR(BfTHR) and Schistosoma mansoni $\mathrm{THR} \alpha(\operatorname{SmTHR} \alpha)$, respectively. Alignment of those sequences indicated that the major functional regions were very conserved (Figure 1 ). So, this clone was named as $S$. japonicum THR $\beta$ (SjTHR $\beta)$. The SjTHR $\beta$ was found to possess a typical nuclear receptor modular motif, including a relatively diverse $\mathrm{N}$-terminal $\mathrm{A} / \mathrm{B}$ domain, a hinge region ( $\mathrm{D}$ domain), a highly conservative $\mathrm{DBD}$, and a moderately conservative C-terminal LBD. The SjTHR $\beta$ DBD possesses two zinc-finger modules (Figure $2 \mathrm{~A}$ ). There is an N-terminal signature sequence which goes before the first zinc-finger module (Figure 1) [2]. In the DNA binding domain, the P-box (CEGCKG) is known to play an important role in determining DNA binding specificity. The P-box in SjTHR $\beta$ is followed by a FFRR

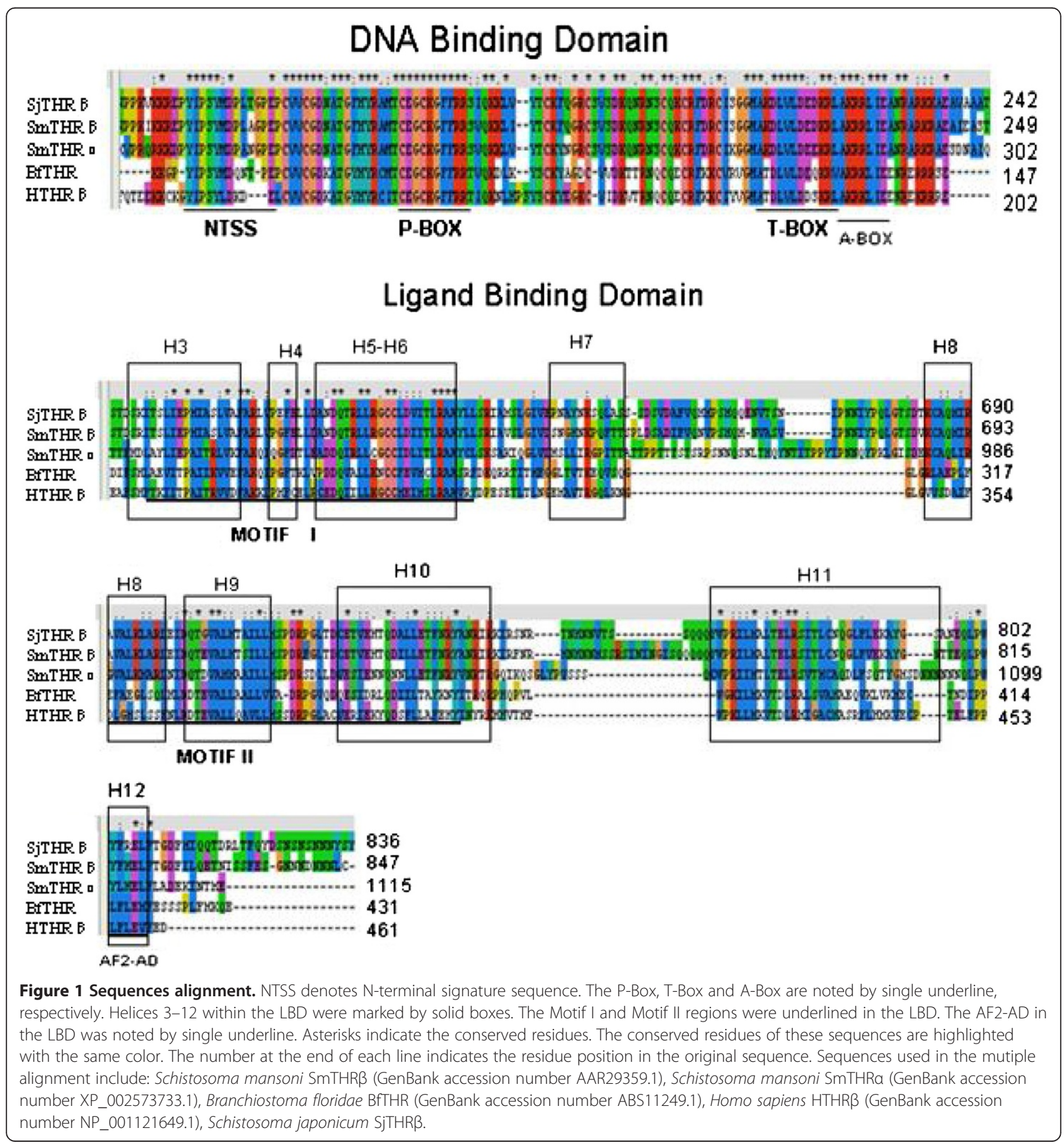




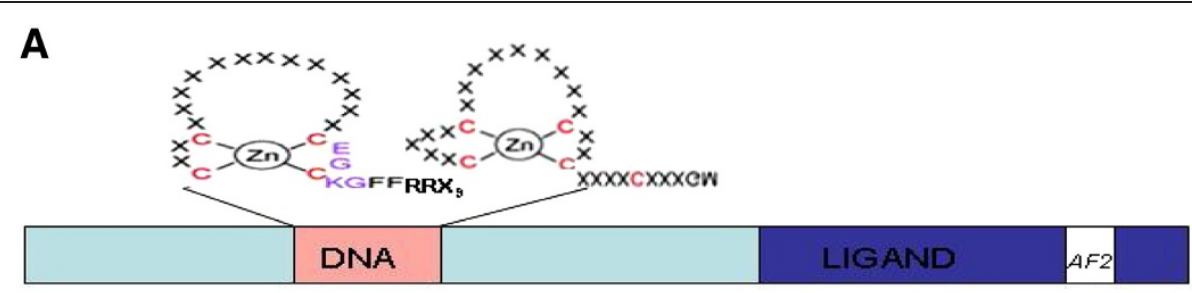

\section{B}

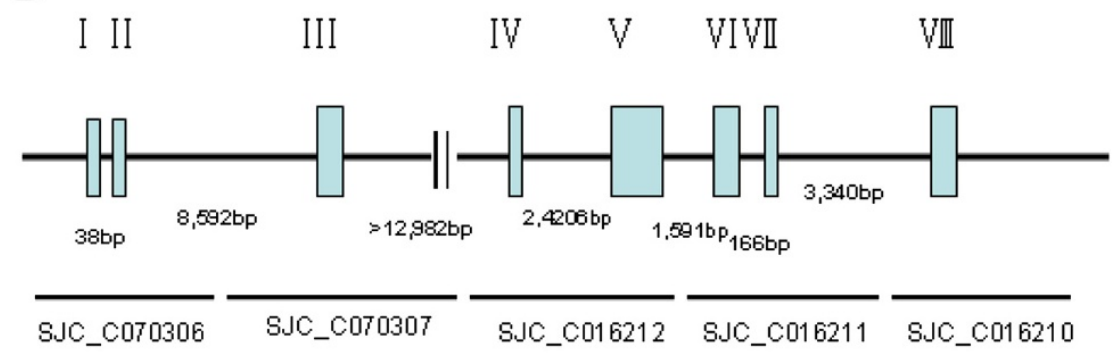

C

\begin{tabular}{|c|c|c|c|c|c|c|}
\hline \begin{tabular}{|l|l|} 
I & II \\
\end{tabular} & III & IV & $\mathrm{V}$ & VI & VII & VIII \\
\hline 5'-UTR & $A / B$ & C & $D$ & & $E$ & $3^{3}$-UTR \\
\hline
\end{tabular}

Figure 2 Gene structure of SjTHR $\beta$. A Two zinc-finger modules of DNA Binding Domain in SjTHR $\beta$ B Exons (roman numerals) and introns are shown with bp sizes indicated. The five genomic supercontigs obtained from the SDSPB genomic sequence database (SJC_C070306,

SJC_C070307, SJC_C016210, SJC_C016211 and SJC_C016212) are shown in the figure. Roman numerals indicate exons. C The amino acid size of exons and their corresponding NR domains are shown. A/B, A/B domain; $C, D N A$ binding domain; $D$, hinge domain; $E$, ligand binding domain.

sequence, which is identical to that of retinoic acid receptors superfamily and is one of the distinguishing features of NR superfamily 1 (Figure 1) [2]. T-box which follows the second zinc finger of the DBD consists of AKDLVLDEDKRL (Figure 1). The T-box plays an important accessory role in forming the dimer interface that determines specificity for the response element recognized [15]. A-box follows the T- box, which is less conserved than either the P-box or T- box. In SjTHR $\beta$, the A-box was AKRRLIE. The LBD of NRs is pivotal for the NR transactivation activity by providing a ligand-binding site, a major dimerization surface and a ligand dependent activation function-2 (AF2-AD) for recruiting cofactors. Like various nuclear receptors in Schistosoma mansoni, the helices 1-2 in the LBD of SjTHR $\beta$ are highly diverse [16]. However, helices 3-12 are relatively conserved. As shown in Figure 1, both helices 3-6 in the motif I [(F, W,Y) $(\mathrm{A}, \mathrm{S}, \mathrm{I})(\mathrm{K}, \mathrm{R}, \mathrm{E}, \mathrm{G}) \mathrm{XXX}(\mathrm{F}, \mathrm{L}) \mathrm{XX}(\mathrm{L}, \mathrm{V}, \mathrm{I}) \mathrm{XXX}(\mathrm{D}, \mathrm{S})$ $(\mathrm{Q}, \mathrm{K}) \mathrm{XX}(\mathrm{L}, \mathrm{V})(\mathrm{L}, \mathrm{I}, \mathrm{F})]$, which corresponds to part of the co-activator binding surface and is the signature of the LBD (Ts), and helices $8-10$ in the consensus motif II. [(EFXXXLXXLXLDXXEKAIXLFSXDRXGLXXXXXVE XLQEXXXXALXXY)] were present with high conservation in SjTHR $\beta$ [14]. In SjTHR $\beta$, the AF2-AD core structure was identified and comprised the sequence YFRELF.

\section{Gene organization analysis}

The cDNA sequence of SjTHR $\beta$ was queried by a BLASTN search against the published $S$. japonicum genomic supercontigs in order to elucidate the $\mathrm{SjTHR} \beta$ gene organization. Five shotgun sequences (SJC_C070306, SJC_C070307, SJC_C016210.

SJC_C016211 and SJC_C016212), which covered the 5'UTR, the entire ORF, and the $3^{\prime}$-UTR, were obtained. It was found that the SjTHR $\beta$ gene spanned more than $32 \mathrm{~kb}$ and consisted of eight exons (bp sizes of 153, 168, 413, 217, 1028, 359,131 and 678) and seven introns (Figure 2B). Comparison with the genomic supercontigs revealed that the A/B domain and $3^{\prime}$-UTR are encoded by one exon. 5'-UTR, DNA binding domain and Hinge domain of SjTHR $\beta$ protein are composed by two exons. The ligand binding domain (LBD) is encoded by three exons. The sizes of each intron were shown in Figure 2B.

\section{Phylogenetic analysis of SjTHR $\beta$}

A phylogenetic tree was constructed based upon the 15 known protein sequences. HRARa, MRARb and SmRXR 
were used as outgroup. The analysis positioned $\mathrm{SjTHR} \beta$ into the nuclear receptor superfamily NR1 and $\mathrm{SjTHR} \beta$ was phylogenetically related to the thyroid hormone receptors. In addition, the SjTHR $\beta$ clustered with the SmTHRs, indicating that SjTHR $\beta$ is most closely related to $\operatorname{SmTHR} \beta$ (Figure 3).

mRNA expression level of SjTHR $\beta$ in the development of schistosomula into adult worms obtained from rabbit Quantitative real-time PCR was performed to evaluate relative mRNA levels of $\mathrm{SjTHR} \beta$ in six different developmental stages. The result revealed that SjTHR $\beta$ mRNA was expressed in $7,13,21,28,35$ and $42 \mathrm{~d}$ worms but with different levels (Figure 4). Interestingly, it showed the highest expression in 21d worms and was much lower in $7 \mathrm{~d}$ and $13 \mathrm{~d}$ schistosomula.

\section{Western blot}

The rSjTHR $\beta$-DBD protein was purified in soluble formation with a molecular weight of about $22 \mathrm{kDa}$, compared to $\mathrm{rSjTHR} \beta-\mathrm{LBD}$ fusion protein in insoluble fraction with a molecular weight of around $45 \mathrm{kDa}$ (Figure 5A lane1 and $\mathrm{B}$ lane5). Polyclonal serum against rSjTHR $\beta$-DBD and $r S j T H R \beta-L B D$ was produced by immunizing mice. The polyclonal anti $\mathrm{rSjTHR} \beta-\mathrm{LBD}$ serum recognized two protein bands in extract from 21 d worms with molecular sizes of approximately $95 \mathrm{kDa}$ and $72 \mathrm{kDa}$ (Figure 5C lane1) and no positive band was observed in extract from E. coli BL21 (DE3). The band of $95 \mathrm{kDa}$ is more apparent than the band of $72 \mathrm{kDa}$. Due to no evidence of two copies of $\operatorname{SjTHR} \beta$ genes, two forms of $\mathrm{SjTHR} \beta$ might result from alternative splicing.

\section{rSjTHR $\beta$-DBD protein binds to Half-site and DRO in vitro}

$\mathrm{rSjTHR} \beta$-DBD protein was over-expressed in $E$. coli and purified. EMSA analysis of rSjTHR $\beta$-DBD-DR0 and Half-site (Hs) interactions was performed in vitro. Gel shift analyses revealed that $\mathrm{rSj}$ THR $\beta$-DBD bound to DR0 and Half-site sequences. Due to DR0 containing one more AGGTCA sequence than Half-site, rSjTHR $\beta$-DBD showed higher affinity to DR0 (Figure 6 A lane2 and B lane3). Formation of the rSjTHR $\beta$-DBD - DR0 and Halfsite complex could be competitively inhibited by addition of excessive unlabeled specific competition probes (SCP) (Figure 6 A lane3 and $\mathrm{B}$ lane2) but not by the non specific competition probe (Non-SCP) (Figure 6 A lane4 and B lane1), suggesting that the binding between the protein and DNAs was specific. Moreover, super-shift experiments showed that anti SjTHR $\beta-D B D$

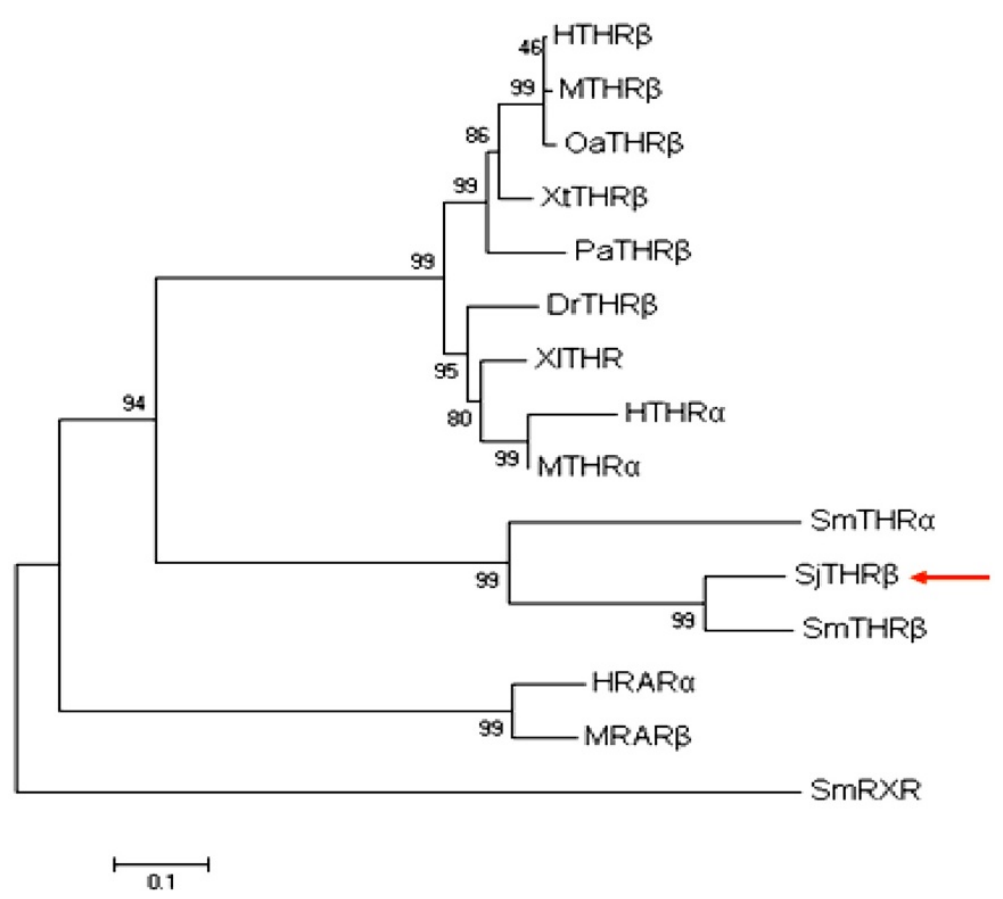

Figure 3 Phylogenetic analysis of SjTHR $\beta$. The phylogenetic tree derived from 15 protein sequences was constructed by the Cluster W program and plotted with MEGA4. The Genbank accession numbers used in the analysis were: HTHRß, Homo sapiens, NP_001121649.1; MTHRß, Mus musculus, AAl19554.1; OaTHRß, Ovis aries, NP_001177320.1; XtTHRß, Xenopus (Silurana) tropicalis, NP_001039270.1; PaTHRß, Pseudopleuronectes americanus, AAV66918.1; DrTHa, Danio rerio, NP_571471.1; XITHR, Xenopus laevis, AAA49969.1; HTHRa, Homo sapiens, NP_001177848.1; MTHRa, Mus musculus, NP_835161.1; SmTHRa, Schistosoma mansoni, XP_002573733.1; SmTHRß, Schistosoma mansoni, AAR29359.1; SjTHRß, Schistosoma japonicum, HRARa, Homo sapiens, P10276; MRARb, Mus musculus, P22605; SmRXR, schistosoma mansoni, AF094759. 


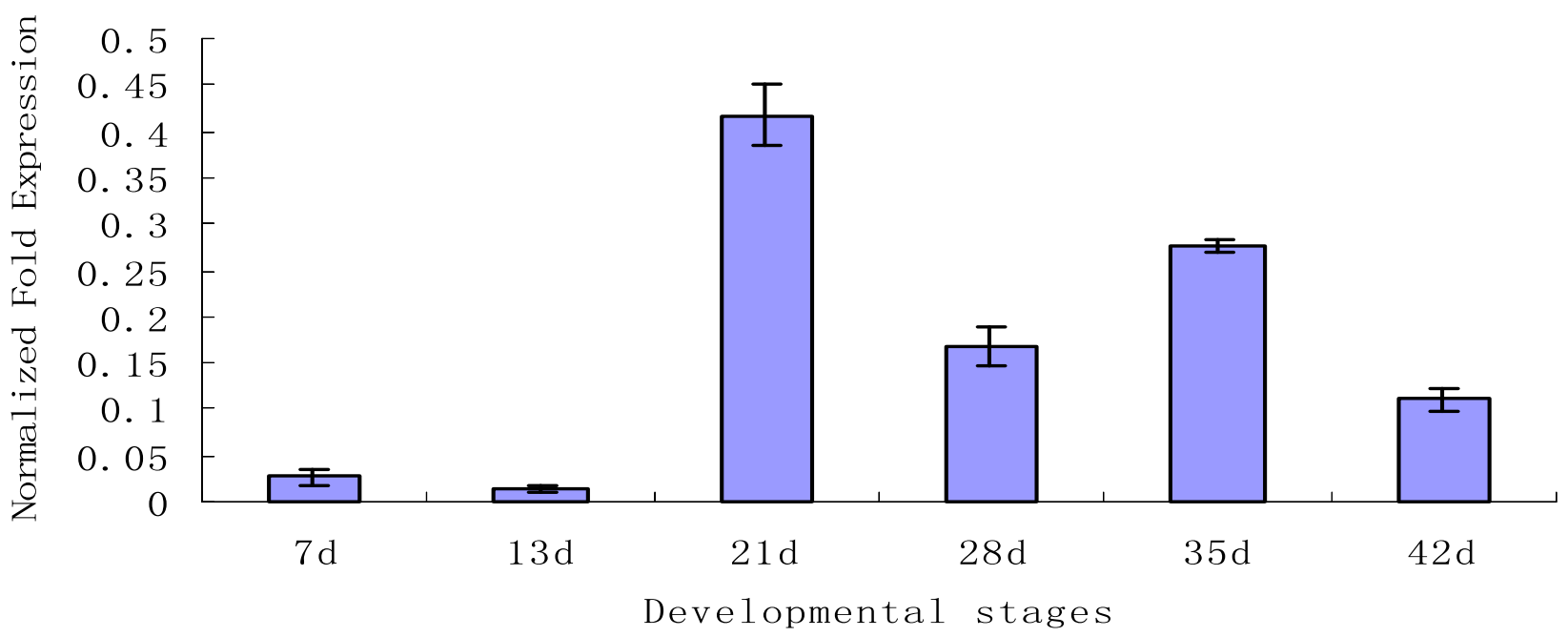

Figure 4 mRNA expression of SjTHR $\boldsymbol{\beta}$ in S. japonicum by real-time quantitative PCR. Data were normalized against the amplified internal housekeeping control gene SjNADH. Worms were collected from infected rabbits at day (d) 7, 13, 21, 28, 35 and 42 days post-infection. This result was obtained by three separate assays with identical protocols. Vertical bars denote the \pm SD about the mean.

serum could partially retard shift of the bands (Figure 6 C lane2 and lane3).

\section{Protective immune efficacy in mice induced by rSjTHR $\beta$-LBD}

In order to evaluate the protection levels induced by $\mathrm{rSj} T H R \beta-\mathrm{LBD}$, the percentage reductions in the worm burden and in the liver egg count were calculated (Table 1). Comparison of mice immunized with rSjTHR $\beta$-LBD to PBS control group, showed a $27.52 \%$ decrease in the worm burden $(\mathrm{P}<0.05)$ and a $29.50 \%$ reduction in egg count $(\mathrm{P}<0.05)$ obtained. The results revealed that $\mathrm{rSjTHR} \beta$-LBD could induce partial protection against $S$. japonicum infection.

\section{Specific antibody detection}

The level of IgG antibody specific to $\mathrm{rSj}$ THR $\beta$-LBD in the sera from both immunized and control mice as detected by ELISA is shown in Figure 7A. In the group of mice immunized with $\mathrm{rSj}$ THR $\beta$-LBD, the specific IgG antibody rose to a higher level after the first injection, thereafter it maintained a high level until the mice were culled. In

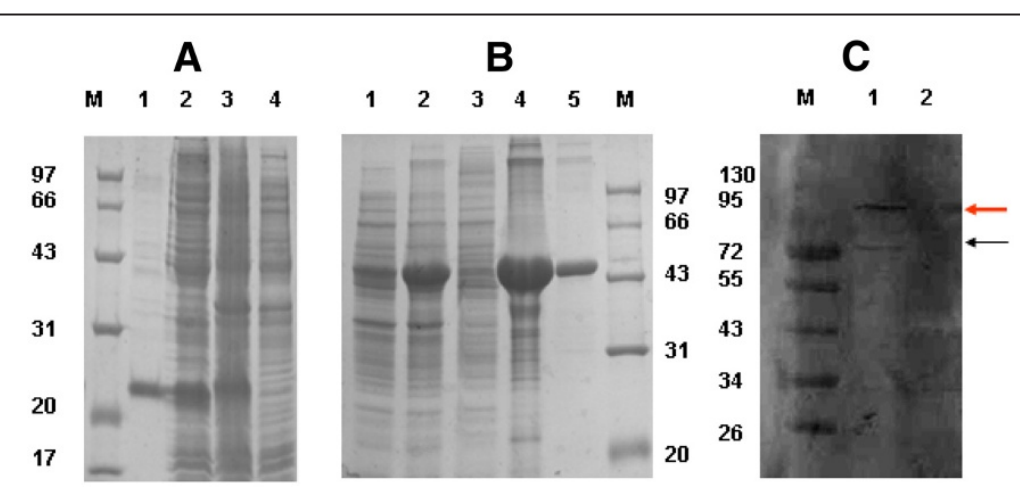

Figure $\mathbf{5}$ Western blot. (A) SDS-PAGE analysis of the rSjTHRß-DBD protein. Lane1: Purified rSjTHRß-DBD protein (about 22 kDa). Lane2: Soluble fractions of expressed product of pET28a (+)-SjTHRß-DBD induced with IPTG of the E. coli extract. Lane3: Expressed product of pET28a (+)-SjTHRßDBD induced with IPTG. Lane4: Expressed product of pET28a (+)-THRß-DBD without induction. (B) SDS-PAGE analysis of the rSjTHRß-LBD protein. Lane1: Expressed product of pET32a (+)-SjTHRß-LBD without induction. Lane2: Expressed product of pET32a (+)-SjTHRß-LBD induced with IPTG. Lane3: Soluble fractions of expressed product of pET32a (+)-SjTHRB-LBD induced with IPTG of the E.coli extract. Lane4: Insoluble fractions of expressed product of pET32a (+)-SjTHRß-LBD induced with IPTG of the E.coli extract. Lane5: Purified rSjTHRß-LBD protein (about 45 kDa) (C) The protein extract of 21d schistosomes (lane1) and E.coli BL21 (DE3) (lane2) were probed with mouse serum specific to rSjTHRB-LBD. The blots are representative of three replicates. M: protein marker. 


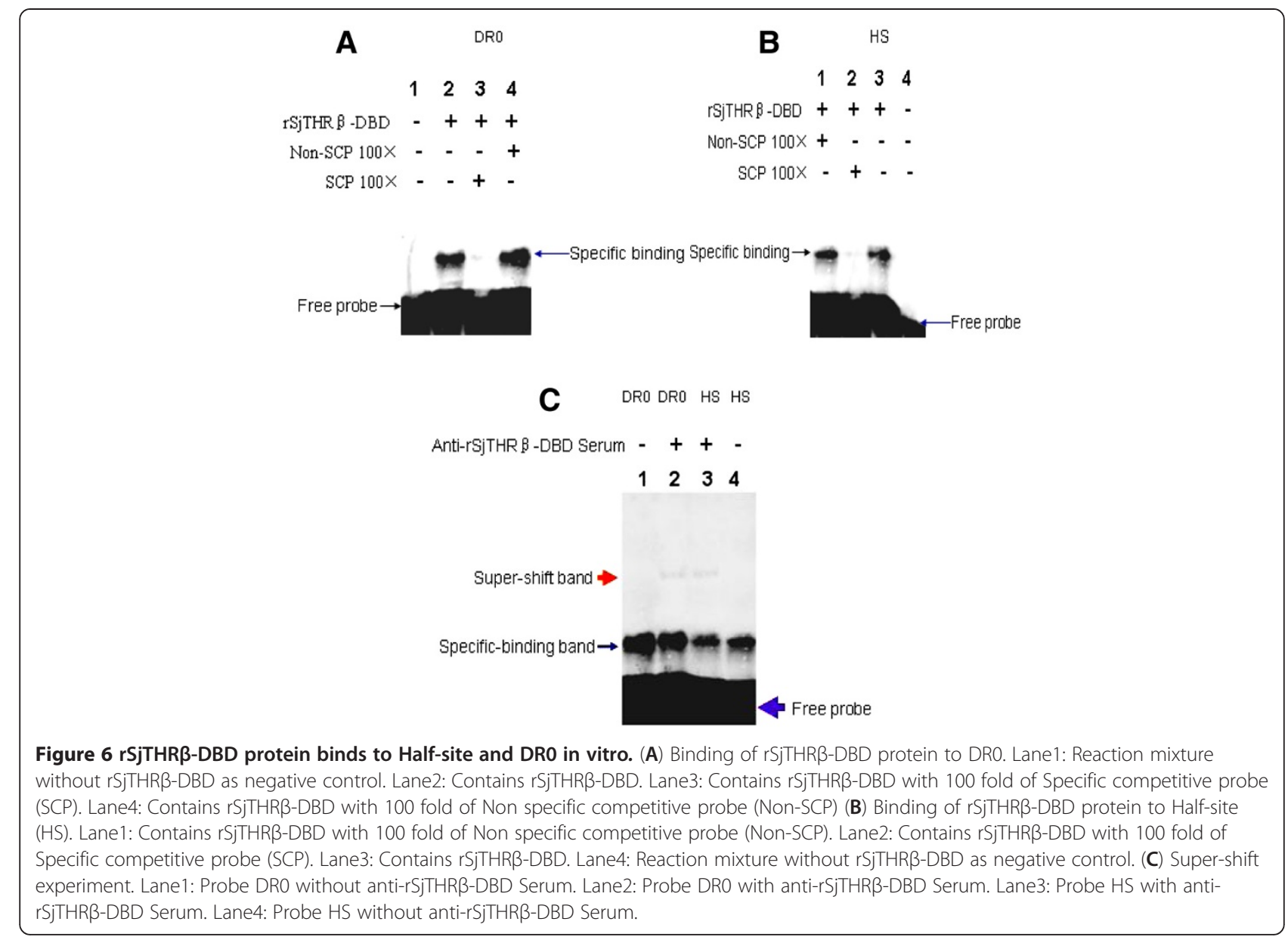

addition, the level of specific IgG1 and IgG2a antibodies induced by rSjTHR $\beta$-LBD was determined (Figure 7B). Both IgG1 and IgG2a levels increased after the first injection, and the ratio of IgG1/IgG2a did not obviously change. The specific IgG, IgG1 and IgG2a levels in both the adjuvant control group and blank control group remained at a lower level until mice were culled.

\section{Cytokine analysis}

IL-2, IL-4, IL-10, TNF- $\alpha$, IL-12 and IFN- $\gamma$ were evaluated by Bio-Plex. As shown in the Figure 8, the level of IL-2, IL-12 and TNF- $\alpha$ in the rSjTHR $\beta$-LBD group were significantly higher than those in the adjuvant control group. The IL-4 and IL-10 levels in the rSjTHR $\beta$-LBD group were slightly higher $(\mathrm{P}>0.05)$ than that in the 206 adjuvant group and there was almost no difference in the IFN- $\gamma$ production between the rSjTHR $\beta$-LBD group and the 206 adjuvant group.

\section{Discussion}

S. japonicum has a complex life cycle including freeliving, aquatic stages and parasitic stages in the intermediate host snail or in the definitive mammalian host and is mainly responsible for intestinal and hepatosplenic schistosomiasis in China, the Philippines, and Indonesia [11]. The genomic information of S. japonicum suggests the existence of an integral hypothalamic-pituitarythyroid axis in this species [17]. TH is synthesized in the thyroid gland under the control of thyroid stimulating hormone (TSH) secreted by the pituitary. TSH

Table 1 Comparison of protective efficacy against S. japonicum challenge in mice induced by SjTHR $\beta$-LBD

\begin{tabular}{ccccc}
\hline group & Average worm burden & worm reduction rate(\%) & Eggs/g liver & Liver egg reduction rate(\%) \\
\hline PBS & $25.25 \pm 4.23$ & - & $77,210.85 \pm 15,437.34$ & - \\
206 & $26.13 \pm 7.99$ & - & $73,623.43 \pm 12,063.51$ & 4.65 \\
rSjTHRß-LBD & $18.3 \pm 4.58$ & $27.52^{*}$ & $54,432.46 \pm 5,341.33$ & $29.50^{*}$ \\
\hline
\end{tabular}

${ }^{*}$ indicate $\mathrm{P}$ value $<0.05$. 

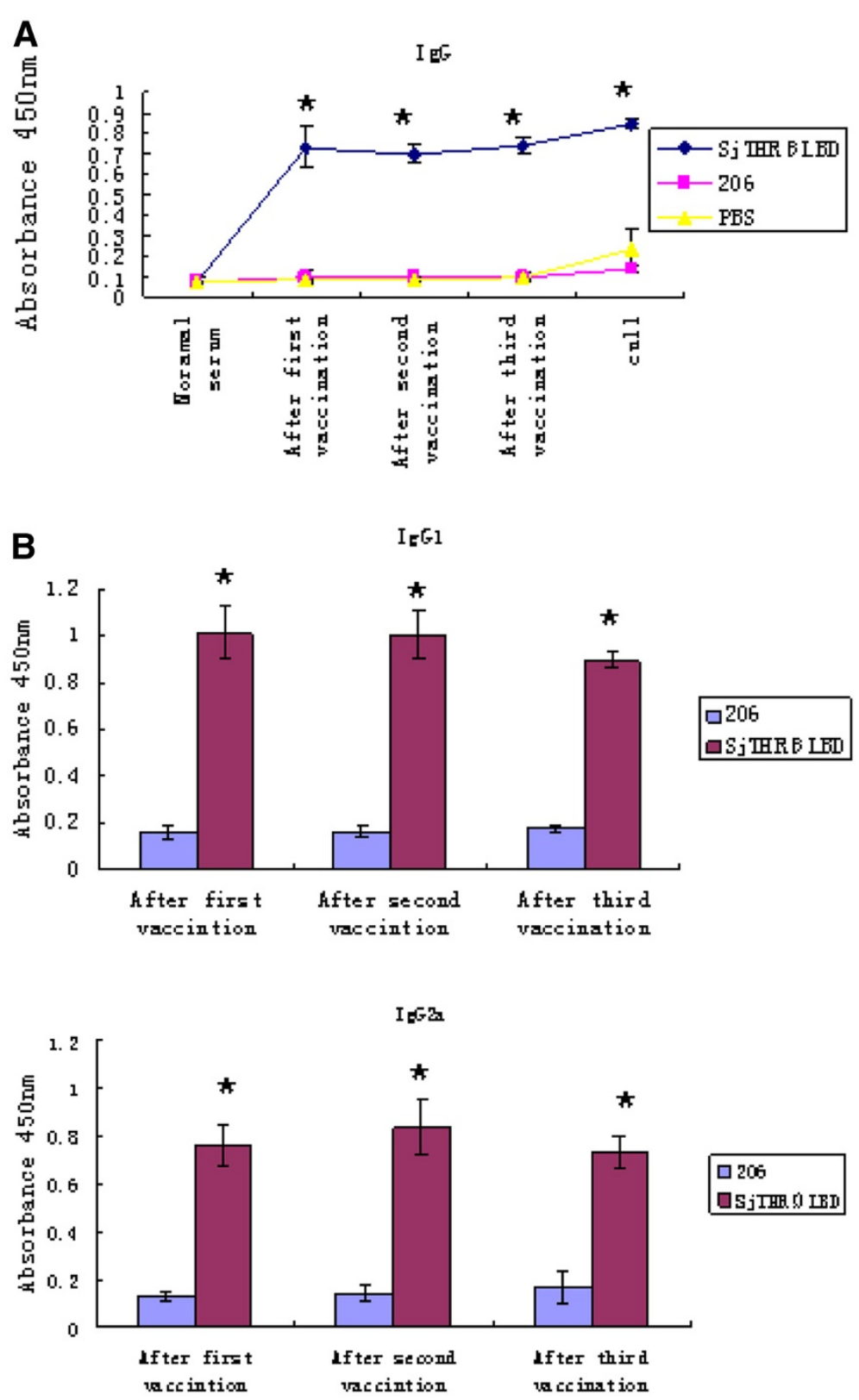

Figure 7 A Detection of specific IgG antibody against rSjTHRß-LBD by ELISA. Mice were injected with rSjTHRß-LBD, 206 adjuvant and PBS, respectively. Figure 7B Detection of specific IgG1 and IgG2a antibodies against rSjTHRß-LBD. This result was obtained by three replicates with identical protocols. Vertical bars depict the \pm SD about the mean. The asterisks $\left(^{*}\right)$ indicate significantly increased serum antibody titers compared with that of the 206 control $(P<0.01)$.

secretion is controlled by thyrotropin-releasing hormone (TRH), which is secreted from the hypothalamus [2]. In addition, TRH, TSH and TH perform their functions by TRH, TSH and TH receptors [17]. Among THRs, two principal THR types (THR $\alpha$ and THR $\beta$ ) with specific physiological functions have been identified from various species, including S. mansoni. According to putative sequences of THR deposited in http://lifecenter.sgst.cn/ schistosoma/cn/schdownload, several pairs of primer were designed to amplify SjTHRs. Based on these primers; we firstly identified a sequence encoding SjTHR $\beta$. However, none of the primers resulted in SjTHR $\alpha$ sequence. The RT-PCR confirmed that SjTHR $\beta$ is constitutively expressed with higher levels from $21 \mathrm{~d}$ and $35 \mathrm{~d}$ worms, suggesting that SjTHR $\beta$ may have a more significant role in the developmental stages of $21 \mathrm{~d}$ and $35 \mathrm{~d}$ worms. The SjTHR $\beta$ contained a highly conserved DBD domain and a moderately conserved LBD domain. Our EMSA show that SjTHR $\beta-$ DBD can bind to a conserved DNA core motif AGGTCA, indicating 

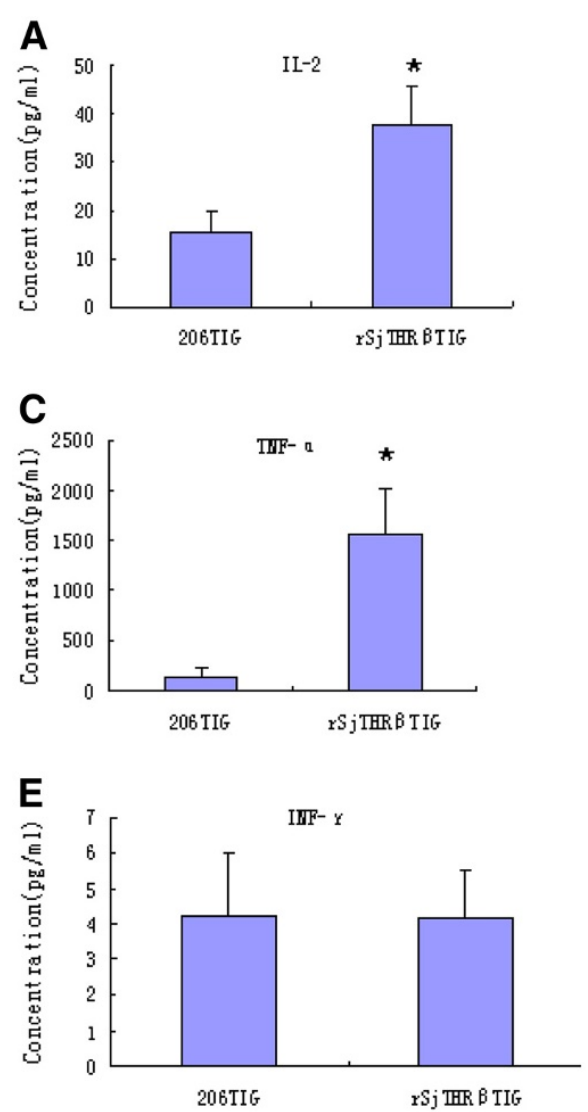
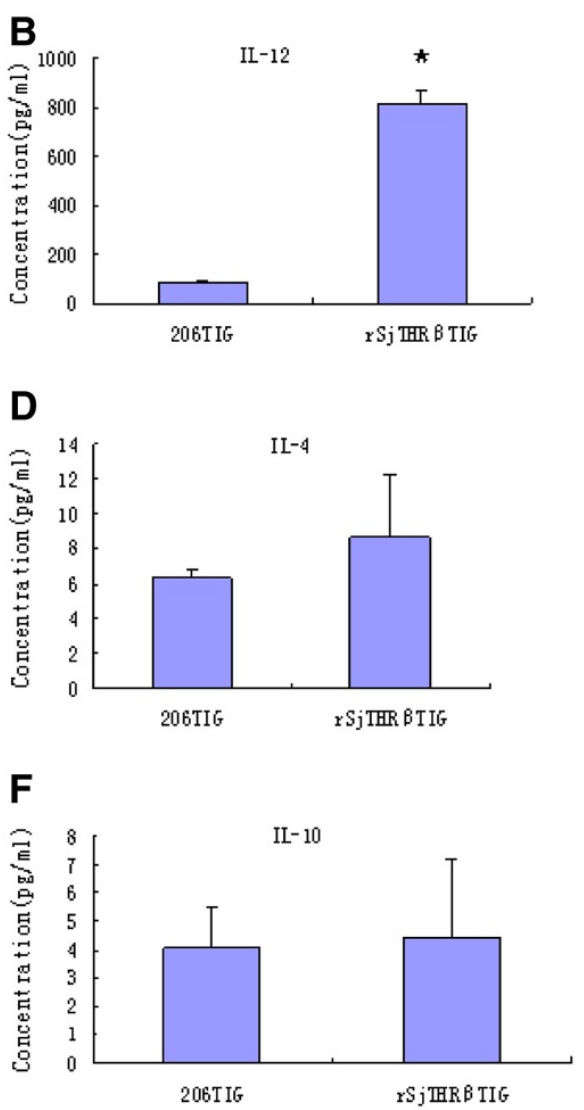

Figure 8 Cytokine analysis between rSjTHRß-LBD and 206 adjuvant group. The level of serum IL-2 (A), IL-12 (B), TNF-a (C), IL-4 (D), INF-Y (E) and IL-10 (F) from mice immunized with rSjTHRß-LBD plus 206 adjuvant or 206 adjuvant only. The asterisk indicates significantly increased serum cytokine level between rSjTHRB-LBD and 206 adjuvant control groups $(p<0.05)$. 206TIG represents the third injection group of 206 adjuvant. rSjTHRßTIG depict the third injection group of rSjTHRß-LBD.

that the function of THR is conserved during evolution. Freebern and his colleagues have proved the binding ability between SmRXR and cis-elements of the P14 gene, which is an eggshell precursor, providing evidence for a possible role in regulation of female-specific gene expression [18]. THR DNA binding is involved in both positive and negative gene regulation [19]. Further identification of the THRE in specific target gene in S. japonicum will help to understand the regulation mechanism of THR.

Even though praziquantel is highly effective in curing schistosomiasis, it still has its limitations that cannot prevent high frequency of re-infection and repair injury [20]. Thus, the discovery of an effective vaccine remains the most potentially powerful means of control for this disease. Previous studies have described the cross-talk of host-parasite relationships between endocrine and immune mediators [21]. The influence of $\mathrm{TH}$ on the immune system is well characterized and could mediate its action on infection. Saule et al. showed that the variation of host TH and interleukin-7 could favor the development of $S$. mansoni [22]. Considering that S. japonicum might have an integral hypothalamic-pituitary-thyroid axis in its genome, we speculate that THR $\beta$ of $S$. japonicum could bind to ligand (TH) of both itself and its host. TH might act through THR, a member of the nuclear receptor superfamily I that intervenes in the expression of numerous genes, on both host and parasite. So, we assumed that SjTHR might have a role in immune regulation during the $S$. japonicum infection process. Here, we cloned and over-expressed SjTHR $\beta$-LBD to evaluate its protective efficacy against schistosoma. In this experiment, rSjTHR $\beta$-LBD could induce partial levels of protection against $S$. japonicum challenge. The mice vaccinated with rSjTHR $\beta$-LBD elicited high levels of specific IgG as well as its subtypes IgG1 and IgG2a antibody via ELISA. IgG2a might predominantly activate $\mathrm{T}$ helper 1 cells, while IgG1 antibody mainly differentiated Th0 precursor in the Th2 direction in mice [23]. Taken together, antibody profiles of vaccinated mice revealed the protective response to be a mixed Th1/Th2 types. Cytokines acting on lymphocytes play an important regulatory and signaling role in the development of an immune response. It is well known that IL-12, together with TNF- $\alpha$, IL- 2 and IFN- $\gamma$ have long been classified as 
a type 1 immune cytokines [24,25]. The presence of IL2 , IFN- $\gamma$, and IL-12 activates the Th0 precursor cells to become Th1 inflammatory T cells. IL-12 gene polarizes the immune responses towards Th- 1 cell development and stimulates the strongest CTL activity [26,27]. In addition, TNF- $\alpha$ is a pro-inflammatory cytokine involved in the cytokine cascade and leukocyte recruitment. On the other hand, IL-4 as well as IL-10 induces a Th0 precursor becoming an armed Th2 helper cell [26]. IL-4 is a prominent Th2 cytokine that plays an important part in stimulating humoral immune responses [28]. Given that the cytokines IL-2, IL-12 and TNF- $\alpha$ in the SjTHR $\beta$ LBD group were significantly enhanced, whereas, the levels of serum IL-4 and IL-10 in the rSjTHR $\beta$-LBD group and 206 adjuvant group were not obviously different, we suggested that $\mathrm{rSjTHR} \beta$-LBD can induce considerably higher levels of Th-1 response enhancing cytokines (IL-2, IL-12 and TNF- $\alpha$ ) than Th-2 response enhancing cytokines (IL-10, IL-4). rSjTHR $\beta$ - LBD vaccination could stimulate a mixed Th1/Th2 type with Th1 dominant in humoral and $\mathrm{T}$ cell responses.

\section{Conclusion}

Herein, we identified SjTHR $\beta$ as the first member of NR superfamily in S. japonicum. Multiple sequence alignment and constructed evolutionary tree showed that SjTHR $\beta$ belongs to nuclear superfamily I. EMSA analysis showed that SjTHR $\beta$-DBD could bind to a conserved DNA core motif, suggesting SjTHR $\beta$ might play an important role in gene regulation in $S$. japonicum. In addition, immunization of BALB/c mice with $\mathrm{rSjTHR} \beta$ LBD vaccination could induce partial protective efficacy against schistosome infection, indicating SjTHR $\beta$ might be a vaccine candidate for schistosomiasis. A full understanding of the role of SjTHR $\beta$ in the biology of $S$. japonicum will add to our knowledge of nuclear receptor gene regulation in schistosomes. Ongoing studies to identify more nuclear receptors involved in host-parasite interactions will aid in mechanistic studies to determine useful drug targets to modulate parasite or host biochemical pathways [29] and treat schistosomiasis.

\section{Competing interests}

The authors declare that they have no competing interests.

\section{Authors' contributions}

Conceived and designed the experiments: $\mathrm{CHQ}, J \mathrm{JL}$. Performed the experiments: $C H Q, M M W, D Z A$. Analyzed the data: $C H Q$. Wrote the paper: $\mathrm{CHQ}$. JJL, CHQ, SFL, YH, ZQF revised the manuscript. All authors read and approved the final manuscript.

\section{Acknowledgements}

We are deeply indebted to Yaojun Shi and Hao Li for helping us to infect rabbits in this work. We are also grateful to Zhixin Zhao for providing laboratory facilities. This work was supported by the National Natural Science Foundation of China (no. 31172315) and Special Fund for Agro-Scientific Research in the Public Interest (no. 200903036).
Received: 12 April 2012 Accepted: 6 August 2012

Published: 13 August 2012

\section{References}

1. Machado DS, Sabet A, Santiago LA, Sidhaye AR, Chiamolera MI, Ortiga-Carvalho TM, Wondisford FE: From the Cover: A thyroid hormone receptor mutation that dissociates thyroid hormone regulation of gene expression in vivo. Proc Natl Acad Sci 2009, 106:9441-9446.

2. Wu W, Niles EG, LoVerde PT: Thyroid hormone receptor orthologues from invertebratespecies with emphasis on Schistosoma mansoni. BMC Evol Biol 2007, 7:150.

3. Velasco LFR, Togashi M, Walfish PG, Pessanha RP, Moura FN, Barra GB, Nguyen P, Rebong R, Yuan C, Simeoni LA, Ribeiro RCJ, Baxter JD, Webb P, Neves FAR: Thyroid Hormone Response Element Organization Dictates the Composition of Active Receptor. J Biol Chem 2007, 282:12458-12466.

4. Zhao WX, Tian M, Zhao BX, Li GD, Liu B, Zhan YY, Chen HZ, Wu Q: Orphan Receptor TR3 Attenuates the p300-Induced Acetylation of Retinoid X Receptor. Mol Endocrinol 2007, 21:2877-2889.

5. Lapa M, Dias B, Jardim C, Fernandes CJ, Dourado PM, Figueiredo M, Farias A, Tsutsui J, Terra-Filho M, Humbert M, Souza R: Cardiopulmonary manifestations of hepatosplenic schistosomiasis. Circulation 2009, 119:1518-1523.

6. Van der Werf MJ, De Vlas SJ, Brooker S, Looman CWN, Nagelkerke NJD, Habbema JDF, Engels D: Quantification of clinical morbidity associated with schistosome infection in sub-Saharan Africa. Acta Trop 2003, 86:125-139.

7. Forrester SG, Warfel PW, Pearce EJ: Tegument expression of a novel type II receptor serine/threonine kinase in Schistosoma mansoni. Mol and Biochem Parasitol 2004, 136:149-156.

8. Lawrence PO: Hormonal effects on insects and other endoparasites in vitro. In Vitro Cell Dev Biol 1991, 27A:487-496.

9. De Mendonça RL, Escrivá H, Bouton D, Laudet V, Pierce RJ: Hormones and Nuclear Receptors in Schistosome Development. Parasitol Today 2000, 16:233-240.

10. Beckage NE: Host-parasite hormonal relationships: a common theme? Exp Parasitol 1991, 72:332-338.

11. Han ZG, Brindley PJ, Wang SY, Chen Z: Schistosoma Genomics: New Perspectives on Schistosome Biology and Host-Parasite Interaction. Annu Rev Genomics Hum Genet 2009, 10:211-240.

12. Wu W, Niles EG, Hirai H, LoVerde PT: Identification and characterization of a nuclear receptor subfamily I member in the Platyhelminth Schistosoma mansoni (SmNR1). FEBS J 2007, 274:390-405.

13. Jones MK, McManus DP, Sivadorai P, Glanfield A, Moertel L, Belli SI, Gobert GN: Tracking the fate of iron in early development of human blood flukes. Int J Biochem Cell Biol 2007, 39:1646-1658.

14. Gobert GN, Moertel L, Brindley PJ, McManus DP: Developmental gene expression profiles of the human pathogen Schistosoma japonicum. BMC Genomics 2009, 10:128.

15. Lu C, Wu W, Niles EG, LoVerde PT: Identification and characterization of a novel fushi tarazu factor 1 (FTZ-F1) nuclear receptor in Schistosoma mansoni. Mol and Biochem Parasitol 2006, 150:25-36.

16. Wu W, Tak EY, LoVerde PT: Schistosoma mansoni: SmE78, a nuclear receptor orthologue of Drosophila ecdysone-induced protein 78 . Exp Parasitol 2008, 119:313-318

17. Liu F, Zhou Y, Wang ZQ, Lu G, Zheng H, Brindley PJ, McManus DP, Blair D, Zhang QH, Zhong Y, Wang S, Han ZG, Chen Z: The Schistosoma japonicum genome reveals features of host-parasite interplay. Nature 2009, 460:345-351.

18. Freebern WJ, Osman A, Niles EG, Christen L, LoVerde PT: Identification of a CDNA encoding a retinoid $X$ receptor homologue Schistosoma mansoni. J Biol Chem 1999, 274:4577-4585.

19. Shibusawa N: Thyroid Hormone Receptor DNA Binding Is Required for Both Positive and Negative Gene Regulation. J Biol Chem 2002, 278:732-738.

20. Bergquist R, Utzinger J, McManus DP: Trick or treat: the role of vaccines in integrated schistosomiasis control. Plos Neglect Trop D 2008, D2:e244

21. Bossu M, Wolowczuk I, Roye O, Saule P, Angeli V, Auriault C, Delacre M, Chassande O: Interleukin-7 at the crossway between endocrine and immune host-parasite relationships in Schistosoma mansoni infection. Curr Trends Immunol 2000, 3:31-44. 
22. Saule P, Adriaenssens E, Delacre M, Chassande O, Bossu M, Auriault C: Early Variation of Host Thyroxine and interleukin-7 Favor Schistosoma mansoni. J Parasitol 2002, 88:849-855.

23. Finkelman FD, Holmes J, Katona IM, Urban JF Jr, Beckmann MP, Park LS, Schooley KA, Coffman RL, Mosmann TR, Paul WE: Lymphokine control of in vivo immunoglobulin isotype selection. Annu Rev Immunol 1990, 8:303-333.

24. Kaplan $\mathrm{G}$, Freedman VH: The role of cytokines in the immune response to tuberculosis. Res Immunol 1997, 147:565-572.

25. Xing Z: The hunt for new tuberculosis vaccines: anti-TB immunity and rational design of vaccine. Curr Pharm Des 2001, 7:1015-1037.

26. Arai KFL, Miyajima A, Miyatake S, Arai N, Yokota T: Cytokines: coordinators of immune and inflammatory responses. Annu Rev Biochem Review. 1990, 59:783-836.

27. Morgan DA, Ruscetti FW, Gallo R: Selective in vitro growth of T lymphocytes from normal human bone marrows. Science 1976, 193:1007-1008.

28. Seder RA, Paul WE: Acquisition of lymphokine-producing phenotype by CD4_T cells. Annu Rev Immunol 1994, 12:635-673.

29. Wang Z, Zhou XE, Motola DL, Gao X, Suino-Powell K, Conneely A, Ogata C, Sharma KK, Auchus RJ, Lok JB, Hawdon JM, Kliewer SA, Xu HE, Mangelsdorf $D J$ : Inaugural Article: Identification of the nuclear receptor DAF-12 as a therapeutic target in parasitic nematodes. Proc Natl Acad Sci 2009, 106:9138-9143.

doi:10.1186/1756-3305-5-172

Cite this article as: Qiu et al: Molecular characterization of thyroid

hormone receptor beta from Schistosoma japonicum and assessment of its potential as a vaccine candidate antigen against schistosomiasis in BALB/c mice. Parasites \& Vectors 2012 5:172.

\section{Submit your next manuscript to BioMed Central and take full advantage of:}

- Convenient online submission

- Thorough peer review

- No space constraints or color figure charges

- Immediate publication on acceptance

- Inclusion in PubMed, CAS, Scopus and Google Scholar

- Research which is freely available for redistribution 